\title{
The Research Progress of Biomimetic Olfaction and Taste Sensors
}

\author{
Ping Wang ${ }^{12^{*}}$, Liujing Zhuang ${ }^{1}$, Zhen Qin ${ }^{1}$,Bin Zhang ${ }^{1}$, Geqiang Gao ${ }^{1}$, Chuanjiang He ${ }^{1}$ \\ ${ }^{1}$ Biosensor National Special Laboratory, Key Laboratory for Biomedical Engineering of Education \\ Ministry, Department of Biomedical Engineering, Zhejiang University, Hangzhou 310027, China \\ 2 State Key Laboratory of Transducer Technology, Chinese Academy of Sciences, Shanghai, China \\ *Corresponding author's e-mail address: cnpwang@zju.edu.cn
}

\begin{abstract}
:
The conventional methods for gas or liquid phase detection and analysis usually rely on the utilization of precision laboratory artificial instrument like chromatography-mass spectrometry (GC-MS). These methods provide an accurate approach for the analysis of type and concentration of the single component in a mixture of substances. However, these methods are time consuming, labor intensive, and expensive. The biomimetic smell and taste sensors provide novel solutions to overcome these drawbacks. In 1982, a novel gas sensing system, electronic nose (e-Nose), was proposed by imitating the sensing mechanisms of biological olfactory systems [1]. In 1990, the first liquid analytical instruments based on non-specific taste sensor array - electronic tongue (e-Tongue) was reported [2]. An e-Tongue using ion selective electrode array and pattern recognition algorithm was developed, which consists of a cross-sensitive chemical sensor array and pattern recognition algorithm and can be used to detect, analyze and identify the complex chemical compositions [3]. The Bioinspired smell and taste sensors (Bio-eNose and Bio-eTongue) mimic the biological chemical sensing mechanisms and could achieve a similar performance to biological olfactory and taste systems by the using of biological functional components for chemical sensing, which show prominent advantages such as high sensitivity, low detection limit, and excellent selectivity[4][5], .
\end{abstract}

Key words: Biomimetic sensors, Olfaction sensors, Taste sensors, Bio-eNose, Bio-eTongue

\section{System Configuration}

The sensitivity, selectivity and response speed of current eNose and eTongue are still far from that of biological olfaction and taste systems. To overcome the development bottleneck of traditional eNose and eTongue, in the in recent years, the concept of bioinspired smell and taste sensors have been increasing recognized as a novel approach. Fig.1 shows the differences in chemical sensing process by biological noses and tongues or electronic noses and tongues, which shows how the chemical signals of Brasil coffee can be detected and recognized. Bio-eNose and Bio-eTongue mimic the biological chemical sensing mechanisms and could achieve a similar performance to biological olfactory and taste systems by using of biological functional components for chemical sensing.

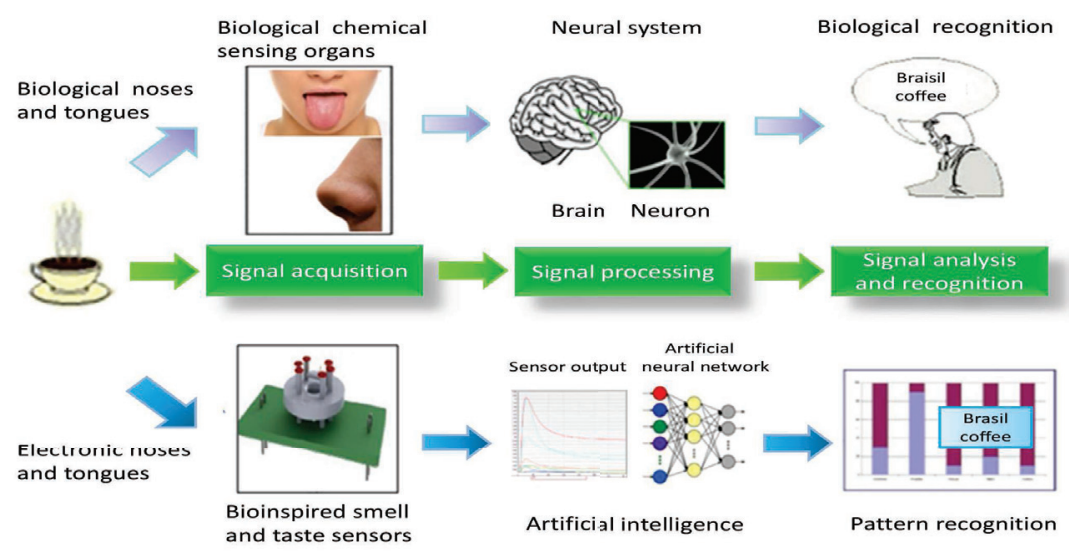

Fig.1 The comparison of Bio-eNose and Bio-eTongue with e-Nose and e-Tongue 


\section{In vitro Bio-eNose and Bio-eTongue}

In intact epithelium, it is possible to estimate the electrochemical potential by keeping the neuronal membrane and environment intact after the epithelium surgically removed. Whereas cells were maintained in their native environment, the acute prepared intact epithelium had several advantages to organotypic function over isolated olfactory receptor neurons or taste cells culture for bioelectronic nose and bioelectronic tongue: The natural states of the neuronal populations of olfactory or taste receptor cells were well preserved. The functional receptor unit of cilia on each receptor cell would not be damaged. Extracellular compartments present in vivo (including supporting cells and basal cells) were preserved. The mucus layer with odor binding protein generated by Bowman's glands and supporting cells were preserved in the olfactory system. The intact epithelium allowed simpler acute

\section{In vivo Bio-eNose and Bio-eTongue}

The in vitro cultured environment leads to shortened cell/tissue survival, so the working life of biosensor is short. In vitro culture would also damage the intact nerve structure of olfactory and taste system and the natural pattern of neuronal activity may be changed. Multiple microelectrode implant technology could overcome these limitations by investigating electrophysiological properties of neuronal populations in vivo. Although this technology causes local tissue damage, it ensures integrity of biological nerve system. The propensity for multi electrode electrophysiological investigation of neuronal populations is well established in both sensory and motor systems. For the in

\section{References}

1. K. Persaud and G. Dodd, Nature, 1982. 299(5881): p. 352-355.

2. K. Hayashi, M. Yamanaka, K. Toko and K. Yamafuji, Sensors and Actuators B, 1990. 2(3): p. 205-213. preparation and easier visualization, without strictly controlled cell culture conditions (i.e. nutrient media, $\mathrm{pH}$, temperature, and listerize). Fig. 2 shows the schematics of a novel bioinspired gustation sensor based on MEA chips and the detected signals in responses to different taste stimuli [5].

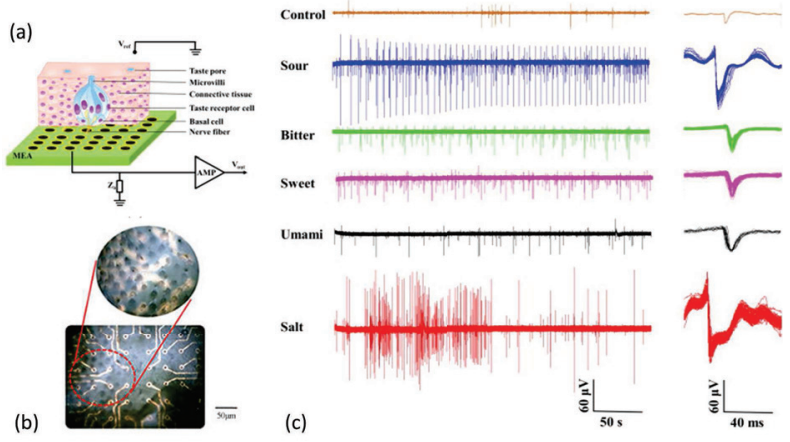

Fig. 2 in vitro Bio-eTongue with taste epithelium accompanied with taste buds and epithelial cells aqnd its extracellular potential stimulated by distinct tastants. (a) Schematic diagram of in vitro biosensing approach using taste epithelium coupled with MEA chip. (b) Image of taste epithelium coupled with MEA chip. (c) Recorded typical responsive electrophysiological signals upon the stimulations of five basic taste qualities.

vivo biosensing system has characteristic of high sensitivity, continuous recording, and specificity, it presents a promising platform for specific trace odorant and tastants detection in real application as shown as in Fig.3 [5].

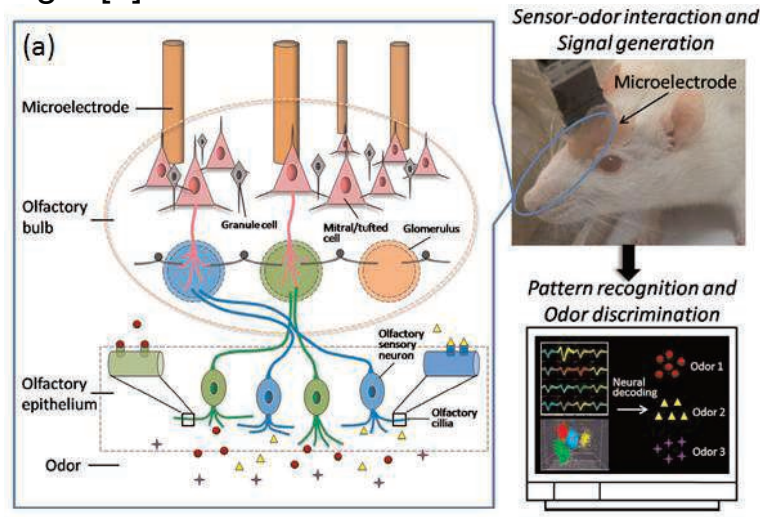

Fig. 3 Sketch map of In vivo Bio-eNose based on implanted electrodes and brain and machine interface (BCl)

3. A.V. Legin, A.M. Rudnitskaya, Y.G. Vlasov, C. Di Natale and A. D'Amico, Sensors and Actuators B, 1999. 58(1-3): p. 464-468.

4. Lim, J.H.; Park, J.; Ahn, J.H.; Jin, H.J.; Hong, S. and Park, T.H, Biosensors and Bioelectronics. 2013, 39, 244-249.

5. Ping Wang, Qingjun Liu, Chunsheng Wu and K.Jimmy Hsia, Springer, Germany, 2015. 